\title{
Differential expression of peroxiredoxin 3 in laryngeal squamous cell carcinoma
}

\author{
Hua Zhang ${ }^{1,2, *}$, Xuexia Liu ${ }^{3, *}$, Lei Chen ${ }^{4, *}$, Li Cai ${ }^{5}$, Ning $\mathrm{Li}^{3}$, Peng Zhu ${ }^{3}$, Jian Chen ${ }^{3}$, \\ Xicheng Song ${ }^{1}$, Guojun $\mathbf{L i}^{2,6}$ \\ ${ }^{1}$ Department of Otolaryngology-Head and Neck Surgery, Yuhuangding Hospital of Qingdao University, Yantai, China \\ ${ }^{2}$ Department of Head and Neck Surgery, The University of Texas MD Anderson Cancer Center, Houston, TX, USA \\ ${ }^{3}$ Department of Central Laboratory, Yuhuangding Hospital of Qingdao University, Yantai, China \\ ${ }^{4}$ Department of Clinical Laboratory, Yuhuangding Hospital of Qingdao University, Yantai, China \\ ${ }^{5}$ Department of Pathology, Yuhuangding Hospital of Qingdao University, Yantai, China \\ ${ }^{6}$ Department of Epidemiology, The University of Texas MD Anderson Cancer Center, Houston, TX, USA \\ *These authors have contributed equally to this work and are considered co-first authors \\ Correspondence to: Xicheng Song, email: songxicheng@126.com \\ Guojun Li, email: gli@mdanderson.org \\ Keywords: peroxiredoxin 3, laryngeal squamous cell carcinoma, Hep-2 cell line, oxidative stress \\ Received: November 10,2016 Accepted: November 22, $2016 \quad$ Published: December 09, 2016
}

\section{ABSTRACT}

Peroxiredoxin (PRDX) proteins are involved in carcinogenesis. PRDX3, which is predominantly localized in mitochondria and up-regulated in several human cancers, seems to confer increased treatment resistance and aggressive phenotypes. This study examined the expression profile of PRDX3 and its possible clinical value in laryngeal squamous cell carcinoma (LSCC). The expression of PRDX3 in LSCC samples was confirmed by Western blotting and further analyzed by immunohistochemistry in LSCC samples of different clinical pathological stages. The results showed that up-regulated expression of PRDX3 was observed in LSCC and associated with poor differentiation ( $\mathbf{P}<\mathbf{0 . 0 1}$ ), primary tumor location, $\mathbf{N}$ category and tumor stage ( $P<0.05)$. Knockdown of PRDX3 in the Hep-2 laryngeal carcinoma epithelial cell line significantly enhanced Hep-2 cells' apoptosis and inhibited their proliferation and migration. Taken together, our results suggest that PRDX3 has substantial clinical impact on the progression of LSCC and shed new light on the role of PRDX3 in treatment resistance and aggressive phenotypes in LSCC.

\section{INTRODUCTION}

Head and neck cancer (HNC) is one of the most malignant carcinomas in the world [1]; $25 \%$ of HNCs are laryngeal carcinomas $[2,3]$, and $95-98 \%$ of these are laryngeal squamous cell carcinoma (LSCC) [4]. According to American Cancer Society data, death from LSCC accounted for $0.7 \%$ of cancer-related deaths in 2009 and its incidence is increasing [5]. Although advances in surgical management, radiotherapy, and chemotherapy have been observed over the last 3 decades, clinical outcomes of LSCC have not significantly improved due to tumor recurrence and metastasis [6]. Despite an improved 5 -year survival rate, $30-40 \%$ of laryngeal carcinoma patients still die of tumor metastasis [3].
In a previous study, we reported increased reactive oxygen species (ROS) levels in LSCC serum, implicating ROS in the development of LSCC [7]. ROS is well known to be involved in the development of cancers [8], and elevated levels of ROS promote cancer development by inducing genomic instability, modifying gene expression, and participating in signaling pathways. However, the nature of this association is both complex and at times paradoxical. A large number of studies have also shown that excessive ROS induce cell apoptosis or autophagy in cells, excessive ROS can cause cell necrosis. Chemotherapy and radiotherapy for cancer may be selectively toxic to tumor cells because they augment oxidant stress and push these already stressed cells beyond their limit [9-11]. 
The degree of oxidant stress in a cell reflects a balance between the rate of ROS production and the activity of scavenging systems that detoxify them. Organisms have developed multiple antioxidant systems to protect against oxidative damage. Peroxiredoxin (PRDX) is the main family of antioxidant proteins. The mammalian PRDXs--PRDX1, PRDX2, PRDX3, PRDX4, PRDX5, and PRDX6--have conserved reactive cysteine residues in their active sites by which hydrogen peroxide $\left(\mathrm{H}_{2} \mathrm{O}_{2}\right)$ is reduced [12]. PRDXs play important roles in cancer development, progression, and recurrence. Recently, the involvement of ROS signaling in tumor metastasis was highlighted $[13,14]$, but the expression profiles of PRDXs in carcinogenesis have not been systematically investigated, especially in LSCC. In the present study, we investigated the expression patterns of PRDXs in LSCC to identify a useful target for the early diagnosis and treatment of LSCC.

\section{RESULTS}

\section{Up-regulated PRDX3 in LSCC tumor tissues}

A protein profile of PRDXs in LSCC was obtained using Western blotting in tumor tissues and normal adjacent tissues from 48 cases of LSCC. As shown in Figure 1, PRDX3 was significantly up-regulated in tumor tissues of LSCC compared with normal adjacent tissues. Significant differences in expression were not observed for the other PRDXs. Subsequently, cellular localization of PRDX3 in LSCC tissues was detected by immunohistochemistry. PRDX3 was mainly expressed in the cytoplasm. Compared to the normal tissues, PRDX3 was dramatically up-regulated in the laryngeal tumor tissues (Figure 2A). The differentiated tissues had different staining intensity (Figure 2B, 2C, 2D). Strong immunostaining (Grade III) was observed in poorly differentiated LSCC samples, moderate immunostaining (Grade II) was observed in moderately differentiated LSCC samples, and weak immunostaining (Grade I) was observed in well-differentiated LSCC samples.

\section{Association of expression of PRDX3 with clinical characteristics and survival}

To investigate the role of PRDX3 in the progression of LSCC, the association between multiple clinical features of LSCC patients and the expression of PRDX3 was analyzed. The clinical features of these patients are provided in Table 1. As shown in Table 2, twenty-three patients (47.9\%) had Grade I, 33.3\% (16 of 48) had Grade II, and $18.8 \%$ (9 of 48) had Grade III. Grade I expression of PRDX3 was also associated with glottic tumor location, lower $\mathrm{N}$ stage and tumor stage (all $\mathrm{P}<0.05)$, while no significant difference in PRDX3 expression was observed for sex, age, tobacco smoking, and T stage $(\mathrm{P}>0.05)$.

Follow-up data for the LSCC patients were available for survival analysis. As shown in Figure 3, the log-rank test revealed a significant difference in survival between the groups with low-moderate (Grade I/II) and high (Grade III) expression of PRDX3 (log-rank, $\mathrm{P}=0.042$ ).

\section{Lower expression of PRDX3 associated with inhibition of proliferation and promotion of apoptosis in Hep-2 cells}

Since PRDX3 expression in LSCC was associated with cell differentiation and tumor depth, the possible biological significance of PRDX3 in tumorigenesis was investigated through loss-of-function studies in vitro.

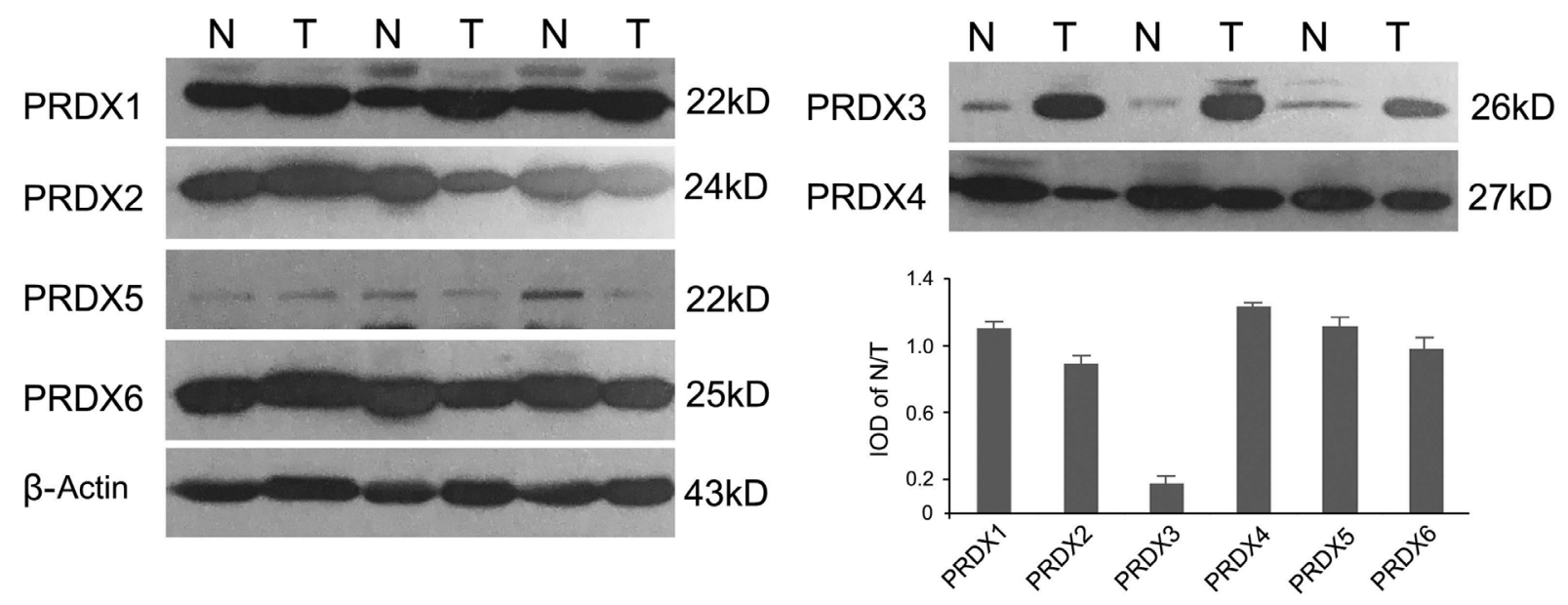

Figure 1: Representative expression profiles of PRDXs in LSCC tissues $(\boldsymbol{n}=\mathbf{4 8})$. Normal adjacent tissues and tumor tissues were analyzed by Western blotting and quantified by integrated optical density (IOD). N: Normal adjacent tissue; T: Tumor tissue. $\beta$-actin expression was used as an internal standard. 
After thePRDX3 expression level was confirmed at 48 $\mathrm{h}$ post-transfection (Figure 4A, 4B), the same number of Hep-2 cells from each group (shRNA-1, shRNA-2, and si-NC group) was inoculated and underwent CCK8 analysis at indicated time points. The result showed knockdown of PRDX3 inhibited the proliferation of Hep2 cells compared with the si-NC transfectants (Figure 4C). In addition, FITC-Annexin V/propidium iodide staining analysis was performed to determine PRDX3induced apoptosis, and the percentage of apoptotic cells significantly increased in PRDX3 knockdown cells when compared to control (Figure 4D). These results indicated that inhibition of PRDX3 expression can inhibit cell proliferation and induce cell apoptosis in vitro.

\section{PRDX3 expression in enhancement of migration in Hep-2 cells}

Migration is an important aspect of tumorigenesis and is closely related to the clinical prognosis. To explore the impact of PRDX3 on LSCC cell migration, a Transwell assay was used to detect the migration ability of Hep-2 cells. As shown in Figure 5, compared with siNC, Hep-2 cells with knockdown of PRDX3 migrated slower, suggesting that knockdown of PRDX3 inhibited the migration ability of laryngeal cancer Hep-2 cells.

\section{DISCUSSION}

Peroxiredoxins, which reduced intracellular peroxides as a novel kind of antioxidant protein, were extensively expressed in various types of cancers and were thought as a biomarker of cancer cells. The role of PRDXs in LSCC is unknown, although growing evidence suggests their involvement in HNC progression and oxidative stress resistance. Yanagawa et al. found that PRDX I expression was associated with local recurrence and lymph node recurrence of squamous cell carcinoma of the tongue. Their results suggested that PRDX I expression indicates tumors with a high potential for recurrence.[15] Ralhan detected 811 proteins as molecular markers in the screening of head and neck squamous cell carcinoma, including the lower expression of PRDX-2 in head and neck squamous cell carcinoma. The lower expression of PRDX-2 suggests that it may be as a tumor suppressor, of course, its mechanism remains to be further research [16]. However, Zhang performed genotyping analyses for tag SNP of PRDX 1, 2 and 6, and then evaluated
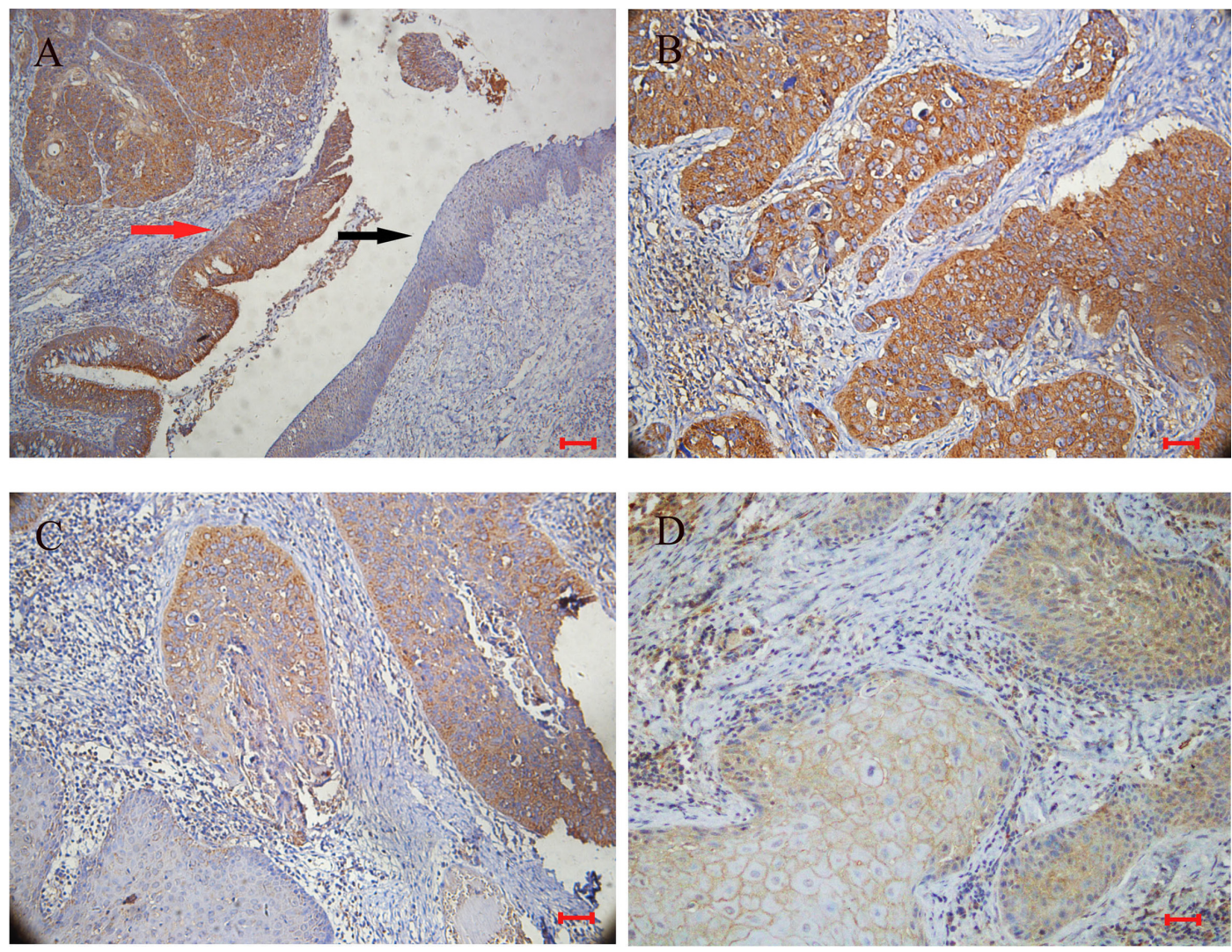

Figure 2: Representative immunohistochemical staining for PRDX3 in sections of LSCC ( $n=48)$. A. Positive staining in cancer tissues (red arrow), and negative staining in adjacent normal epithelium (black arrow) $(\times 100$; bar $=100 \mu m)$; B. strong immunostaining in poorly differentiated LSCC samples $(\times 200$; bar $=50 \mu \mathrm{m})$; C. moderate immunostaining in moderately differentiated LSCC samples $(\times 200$; bar $=50 \mu \mathrm{m})$; D. weak immunostaining in well-differentiated LSCC samples $(\times 200$; bar $=50 \mu \mathrm{m})$. 
Table 1: Characteristics of LSCC patients $(n=48)$

\begin{tabular}{|c|c|}
\hline Variable & $\mathbf{N}(\%)$ \\
\hline No. of patients & 48 \\
\hline \multicolumn{2}{|l|}{ Age } \\
\hline Median age & 65 \\
\hline Range & $41-81$ \\
\hline \multicolumn{2}{|l|}{ Sex } \\
\hline Male & $45(93.8)$ \\
\hline Female & $3(6.2)$ \\
\hline \multicolumn{2}{|l|}{ Tobacco smoking } \\
\hline Ever & $44(91.7)$ \\
\hline Never & $4(8.3)$ \\
\hline \multicolumn{2}{|l|}{ Primary tumor location } \\
\hline Supraglottic cancer & $9(18.8)$ \\
\hline Glottic cancer & $36(75.0)$ \\
\hline Infraglottic cancer & $3(6.2)$ \\
\hline \multicolumn{2}{|l|}{ TNM stage } \\
\hline \multicolumn{2}{|l|}{ T-category } \\
\hline $1-2$ & $26(54.2)$ \\
\hline $3-4$ & $22(45.8)$ \\
\hline \multicolumn{2}{|l|}{ N-category } \\
\hline 0 & $36(75.0)$ \\
\hline $1-3$ & $12(25.0)$ \\
\hline \multicolumn{2}{|l|}{ M-category } \\
\hline 0 & $48(100.0)$ \\
\hline 1 & $0(0)$ \\
\hline \multicolumn{2}{|l|}{ Tumor stage } \\
\hline I-II & $23(47.9)$ \\
\hline III-IV & $25(52.1)$ \\
\hline \multicolumn{2}{|l|}{ Histological grade } \\
\hline G1 & $23(47.9)$ \\
\hline $\mathrm{G} 2$ & $16(33.3)$ \\
\hline G3 & $9(18.8)$ \\
\hline G4 & $0(0)$ \\
\hline \multicolumn{2}{|l|}{ Treatment } \\
\hline Surgery only & $30(62.5)$ \\
\hline Surgery + Adjuvant TX ${ }^{\mathrm{a}}$ & $16(33.3)$ \\
\hline Others ${ }^{b}$ & $2(4.2)$ \\
\hline
\end{tabular}

a Adjuvant TX: adjuvant radiotherapy and/or chemotherapy.

${ }^{\mathrm{b}}$ Others: palliative treatment and/or treatment at outside institution. 
Table 2: Association between PRDX3 expression and clinicopathological characteristics in LSCC patients

\begin{tabular}{|c|c|c|c|c|}
\hline \multirow{2}{*}{ Variable } & \multicolumn{3}{|c|}{ PRDX3 expression } & \multirow{2}{*}{$P$ value } \\
\hline & Grade I & Grade II & Grade III & \\
\hline \multicolumn{5}{|l|}{ Age } \\
\hline$\leq 65$ years & 12 & 9 & 4 & 0.929 \\
\hline$>65$ years & 11 & 7 & 5 & \\
\hline \multicolumn{5}{|l|}{ Sex } \\
\hline Male & 21 & 15 & 9 & 1.000 \\
\hline Female & 2 & 1 & 0 & \\
\hline \multicolumn{5}{|l|}{ Tobacco smoking } \\
\hline Ever & 20 & 15 & 9 & 0.657 \\
\hline Never & 3 & 1 & 0 & \\
\hline \multicolumn{5}{|l|}{ Primary tumor location } \\
\hline Supraglottic cancer & 2 & 3 & 4 & 0.031 \\
\hline Glottic cancer & 20 & 12 & 3 & \\
\hline Infraglottic cancer & 1 & 1 & 2 & \\
\hline \multicolumn{5}{|l|}{ T-category } \\
\hline $1-2$ & 15 & 8 & 3 & 0.245 \\
\hline $3-4$ & 8 & 8 & 6 & \\
\hline \multicolumn{5}{|l|}{ N-category } \\
\hline 0 & 21 & 10 & 5 & 0.032 \\
\hline $1-3$ & 2 & 6 & 4 & \\
\hline \multicolumn{5}{|l|}{ Stage } \\
\hline I-II & 16 & 5 & 2 & 0.017 \\
\hline III-IV & 7 & 11 & 7 & \\
\hline \multicolumn{5}{|l|}{ Histological grade } \\
\hline G1 & 23 & 0 & 0 & 0.000 \\
\hline G2 & 0 & 16 & 0 & \\
\hline G3 & 0 & 0 & 9 & \\
\hline
\end{tabular}

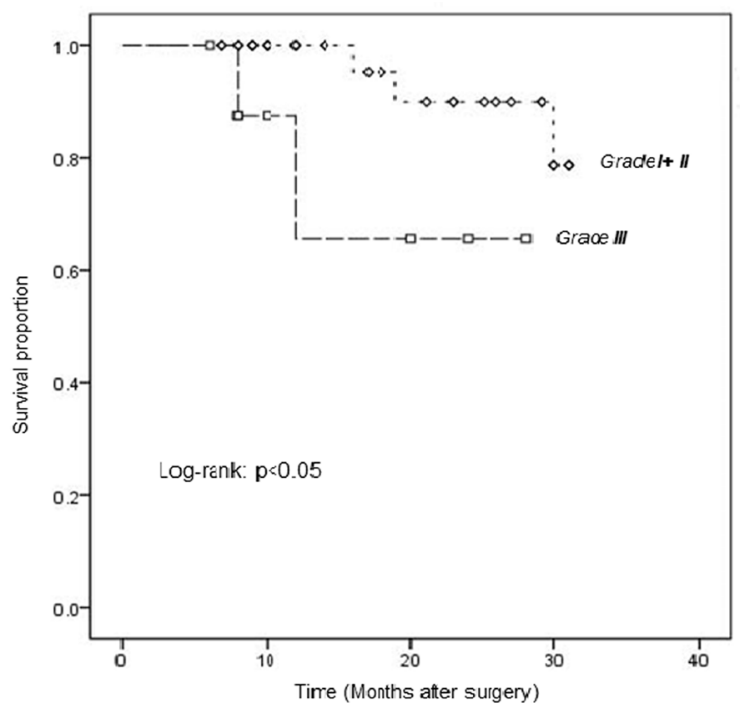

Figure 3: Kaplan-Meier analysis of survival in LSCC patients, stratified according to PRDX3 expression. Comparison of overall survival curves for patients with low-moderate (Grade I/II) and high (Grade III) PRDX3 expression in tumors ( $\mathrm{P}=0.042)$. 
the association with susceptibility and clinic stage of esophageal squamous cell carcinoma (ESCC) in a casecontrol study. Their data suggested that polymorphisms of PRDX 1, 2 and 6 were not associated with esophageal cancer [17]. Our previous study implicated antioxidant proteins in laryngeal carcinoma [7]; thus, the present study investigated the expression profiles and functions of the antioxidant protein family PRDX in laryngeal carcinoma.

All PRDX proteins were found to be expressed in LSCC tumors and adjacent normal tissues, but PRDX3 was significantly up-regulated in tumor tissues, indicating that it may serve as a promising tumorigenesis-associated protein. Therefore, its roles in laryngeal tumorigenesis deserve to be further studied. The immunohistochemistry results were consistent with those of Western blotting and showed PRDX3 was mainly expressed in cytoplasm. The expression of PRDX3 was converse to the degree of histological differentiation: strong immunostaining was observed in poorly differentiated LSCC samples and weak immunostaining in well-differentiated samples.

Most of the patients in our study had early laryngeal cancer, and most of the early laryngeal carcinoma tissue
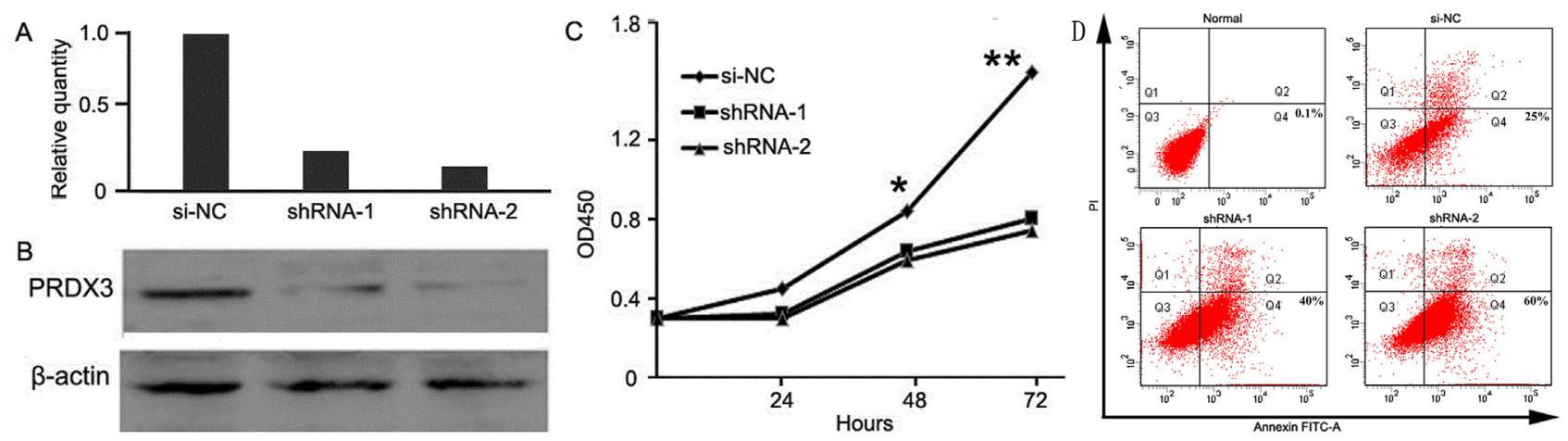

Figure 4: Cell assays of knockdown of PRDX3 in Hep-2 cells. Knockdown effect was verified by quantitative RT-PCR A. and Western blotting B. C. Cell growth was evaluated by Cell Counting Kit- $8\left({ }^{*} \mathrm{p}<0.05\right.$ and $\left.* * \mathrm{P}<0.01\right)$. D. Cell apoptosis assay was detected by flow cytometry. Normal Hep-2 cells without treatment were used as negative control. Hep-2 cells transfected with si-NC were used as positive control. Apoptosis rates were $0.1 \%, 25 \%, 40 \%$, and $60 \%$, respectively.
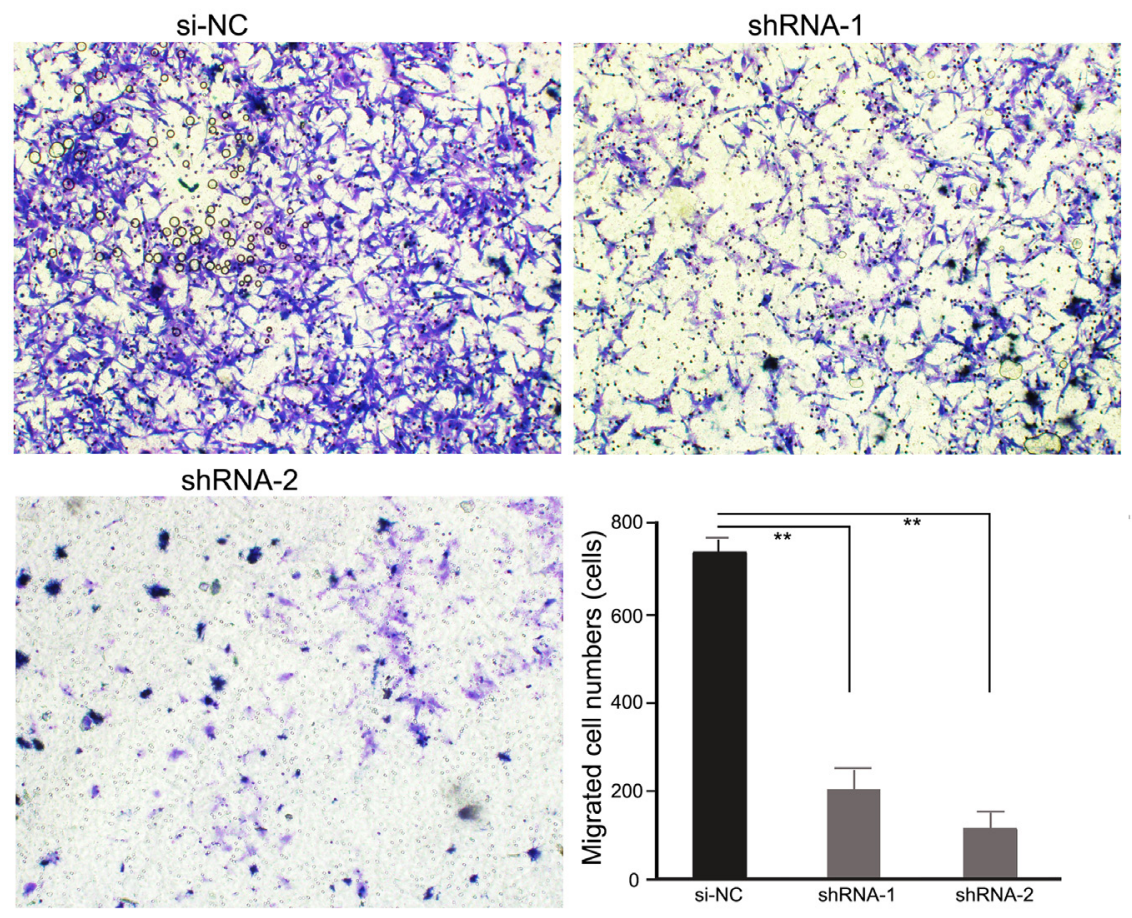

Figure 5: Transwell assays of Hep-2 cells transfected with PRDX3 shRNAs. Representative images (magnification, $\times 100$ ) showed the migration of shRNA-transfected Hep-2 cells. The statistical graph indicates the means \pm SEM of the number of cells from 8 random high-power fields (magnification, $\times 200$ ) counted from three independent experiments. 
differentiation degree was better. So samples with Grade I expression in our study were the maximum and Grade III were the minimum. We found a significant difference in PRDX3 expression according to $\mathrm{N}$ category, tumor stage, and histological grade $(\mathrm{p}<0.05)$. We also found a difference of PRDX3 expression in primary tumor location. Just because the glottic cancer was the most common in all laryngeal carcinoma, and moderate, high differentiation were the majority in it. In all cases, weak PRDX3 expression was associated with less-advanced disease and strong expression was associated with moreadvanced disease.

The survival curves showed that the prognosis of patients with PRDX3 expression of Grade I+II was better than those of Grade III $(p<0.05)$. It showed that the patients with higher differentiation degree had better prognosis. Maybe we need more cases and longer followup to confirm the results further.

PRDX3 was mainly located in mitochondria. Because of the active and indefinite growth of cancer cells and relatively low supply of oxygen, ROS production from mitochondria is accordingly increased [18]. Increased mitochondrial ROS generation and the disturbance of antioxidant systems in cancer cells may lead to oxidative stress and a hypoxic microenvironment, subsequently leading to the induction of apoptosis [19]. Since mitochondrion is the main apoptotic mediator, it is imaginable that control of the ROS level by PRDX3 is involved in apoptotic inhibition. In fact, accumulating studies have suggested that PRDX3 plays an active inhibitory role in chemical-induced oxidation and subsequent apoptosis [19-21]. In the present study we hypothesized that PRDX3 may also be involved in laryngeal tumorigenesis through affecting cell proliferation or apoptosis. We selected the laryngeal tumor cell line Hep-2 to perform in vitro functional analyses by constructing knockdown vectors of PRDX3. CCK8 assay showed that knockdown of PRDX3 significantly inhibited the growth of Hep-2 cells. Meanwhile, the down-regulation of PRDX3 could significantly promote cell apoptosis. Migration is an important aspect of tumorigenesis and is closely related to cell proliferation. Transwell assays indicated that knockdown of PRDX3 significantly inhibits Hep-2 cell migration, which may be due to an increase in apoptosis owing to downregulation of PRDX3. The combined results indicated that PRDX3 is a key protein involved in laryngeal tumorigenesis. Since most chemotherapy or radiotherapy for cancer works through an ROS increase and apoptotic induction, our results provide a clue that PRDX3 may be involved in therapy resistance in LSCC.

In conclusion, the present study identified the protein profiles of the PRDX family in LSCC and showed that down-regulation of PRDX3 in Hep- 2 cells induced cell apoptosis and inhibited cell proliferation and cell migration. The detailed roles and mechanisms of down- regulated expression of PRDX3 in LSCC warrant further study, and the present study indicates that PRDX3 may be a potential molecular target for novel targeted therapy against LSCC. We will collect more clinical cases and extend the follow-up period to validate PRDX3's roles in clinical diagnosis or assessment of prognosis.

\section{MATERIALS AND METHODS}

\section{Tissue samples}

Tissue samples of LSCC tumors and adjacent healthy tissue were collected from 48 patients who were treated at Yuhuangding Hospital of Qingdao University, Department of Otorhinolaryngology Head and Neck Surgery from 2014 to 2016. The clinical features of these patients are summarized in Table 1. The patients were newly diagnosed with LSCC and had not received any treatment prior to biopsy. The normal tissues adjacent to the tumors were collected at sites more than $2 \mathrm{~cm}$ from the edge of the tumor mass and used as a control group. The study protocol was approved by the Committee of Ethics in Research of Yuhuangding Hospital of Qingdao University. All samples were obtained after informed consent was received from the patients. Tumor stage and primary tumor location were defined according to the seventh edition of laryngeal cancer staging international standards revised by the Union for International Cancer Control in 2009 [22]. The primary therapy was surgery in 30 cases, surgery combined with adjuvant radiotherapy and/or chemotherapy in 16 cases, and palliative care and/ or treatment at an outside institution in 2 cases. All 48 patients underwent follow-up ranging from 6 to 32 months (mean: 18.9 months).

\section{Cell line}

The LSCC cell line Hep-2 was purchased from the Cell Bank of the Chinese Academy of Medical Sciences (Shanghai, China). Cells were cultured in 6-well plates with a density of $1 \times 10^{6}$ cells $/ \mathrm{ml}$. Cells were cultured with high-glucose DMEM medium (Sigma-Aldrich, St. Louis, MO, USA) supplemented with $10 \%$ fetal bovine serum (Gibco BRL, Waltham, MA, USA) in $5 \% \mathrm{CO}_{2}$ in a humidified atmosphere at $37^{\circ} \mathrm{C}$.

\section{Protein extraction}

For protein extraction, tissue samples were frozen in liquid nitrogen and ground into powder. The powder was then collected and dissolved in lysis buffer. Cultured cells were washed twice with cold phosphate-buffered saline, collected, and dissolved in lysis buffer. After sonicated for 2 min separately, samples were allowed to rest at $4^{\circ} \mathrm{C}$ for $2 \mathrm{~h}$. Centrifugation at $12000 \times \mathrm{g}$ for 45 min at $4^{\circ} \mathrm{C}$ was then performed. After centrifugation, the 
supernatant was collected and its protein concentration was measured. Each protein sample was then stored at $-80^{\circ} \mathrm{C}$ until use.

\section{Western blotting}

Western blotting was performed as described previously [23]. Proteins were separated by $12 \%$ gel SDS-PAGE, transferred to polyvinylidene difluoride membranes, blocked with 5\% (w/v) skim milk for $1 \mathrm{~h}$, and co-incubated for $1 \mathrm{~h}$ with the primary antibody of PRDX1 (ab41906, Abcam, Cambridge, UK), PRDX2 (sc-23967, Santa Cruz Biotechnology, USA), PRDX3 (YT3873, ImmunoWay, Plano, TX, USA), PRDX4 (ab59542, Abcam, Cambridge, UK), PRDX5 (ab140926, Abcam, Cambridge, UK), or PRDX6 (sc-134478, Santa Cruz Biotechnology, USA) at room temperature with gentle agitation. The membranes were washed with $0.5 \%(\mathrm{v} / \mathrm{v})$ Tween-20 in Tris-buffered saline (TBS) 3 times and then incubated with horseradish peroxidaseconjugated anti-IgG for $1 \mathrm{~h}$ at room temperature. The immune-reactive complexes were detected using an enhanced chemiluminescence (ECL) kit (Amersham Life Sciences, Marlborough, MA, USA). $\beta$-actin (sc-81178, Santa Cruz Biotechnology, USA) was used as an internal standard. The images were analyzed by commercial image analysis software (Gene Tools, version 4.02; Syngeneic, Cambridge, UK). The integrated optical density (IOD) of positive immunostaining was calculated, and the IOD ratio of target protein to $\beta$-actin was used to express the results of the Western blot analysis.

\section{Immunohistochemical staining}

Paraffin-embedded tumor tissues and normal adjacent tissues were obtained from Yuhuangding Hospital of Qingdao University. Four-micrometer-thick sections were de-waxed, and then put in $0.01 \mathrm{M}$ citrate buffer $(\mathrm{PH}=6.0)$ for antigen retrieval by heating in a microwave oven for $15 \mathrm{~min}$. Endogenous peroxidases of sections were inhibited by incubation with $3 \%(\mathrm{v} / \mathrm{v}) \mathrm{H}_{2} \mathrm{O}_{2}$ for 10 min. Then $3 \%(\mathrm{w} / \mathrm{v})$ bovine serum albumin in TBS was used to block non-specific binding with antibodies at room temperature for $1 \mathrm{~h}$. Sections were then incubated with PRDX3 overnight at $4^{\circ} \mathrm{C}$. After the sections were washed with TBS several times, they were incubated with horseradish peroxidase conjugated anti-rabbit IgG (ZhongShan Biotechnology, Zhongshan, Guangdong, China) at a final dilution of $1: 200$ for $0.5 \mathrm{~h}$ at $37^{\circ} \mathrm{C}$. Diaminobenzidine (DAB) kit (Zhong-Shan Biotechnology) was used to visualize the peroxidase activity at binding sites. Hematoxylin was used to counterstain the sections. Then the sections were dehydrated and mounted for brightfield microscopy (DM LB2, Leica, Wetzlar, Germany). Pre-immune rabbit IgG was used as a negative control. The intensity of the staining was classified into 3 groups as follows: Grade I, weak staining; Grade II, moderate staining; and Grade III, strong staining.

\section{Plasmids, shRNAs, and transfection}

Two PRDX3 shRNAs (shRNA-1 and shRNA-2) and negative control shRNA (si-NC) were purchased from Shanghai Sangon Biotechnology Company, Shanghai, China. The sequences of shRNA targeting the PRDX3 transcript were as follows: shRNA-1 5'- GATCCTGG AGTCATCAAGCATTTGAGCGTCTTCAAGAGAG ACGCTCAAATGCTTGATGACTCCATTTTTTA -3'; shRNA-2 5'-GATCCTTATTCAGCACCAGTTCCTCA TGCCTTCAAGAGAGGCATGAGGAACTGGTGCTG AATAATTTTTTA -3'. The PRDX3 gene was amplified using polymerase chain reaction (PCR) with the primers, each containing EcoR I and Xho I sites. The PCR products were digested with EcoR I and Xho I and inserted into a pEGFP-C3 vector. When Hep-2 cells that were seeded onto 6-well plates grew to $70 \%$ confluence, the plasmids were transfected into these cells using X-TREME GENE HP DNA Transfection Reagent (Roche, Mannheim, Germany). At indicated time points after the transfection, cells were harvested for further analysis.

\section{Reverse transcription-polymerase chain reaction (RT-PCR)}

RNA of Hep-2 cells with transfection of si-NC or 2 PRDX3 shRNAs were extracted with Tri-zol reagent (Invitrogen, Grand Island, NY, USA) following the manufacturer's instructions. RNA (1ug) was reverse transcribed with 2 U Avian Myeloblastosis Virus Reverse Transcriptase (Promega, Madison, WI, USA), as described by the manufacturer. The primer sequences for PRDX3 were: forward, 5'-GTTGTCGCAGTCTCAGTGG-3'; reverse, 5'-GACGCTCAAATGCTTGATG-3'. $\beta$-actin (forward, 5'-ACGTTGACATCCGAAAGACC-3'; reverse, 5'-CCACCGATCCACACAGAGTA-3') was used as the internal control. PCR products were analyzed by Gene Tools (Syngene, Frederick, MD, USA). Results were shown as the relative quantity of target genes per $\beta$-actin. Deionized water was used in place of $\mathrm{cDNA}$ as a negative control.

\section{Cell proliferation assay}

Hep-2 cells with transfection of si-NC or 2 PRDX3 shRNAs were seeded at $1 \times 10^{3}$ cells/well in 96 -well plates. Cell viability was assayed by using cell counting kit (CCK)-8 (Dojindo, Kumamoto, Japan) according to the manufacturer's protocols. Briefly, CCK8 reagents were added to the wells at indicated time points and incubated at $37^{\circ} \mathrm{C}$ for $2 \mathrm{~h}$. The absorbance at $450 \mathrm{~nm}$ was recorded with a micro-well plate reader (Synergy TM HT, BIOTEK, Winooski, VT, USA). 


\section{Cell apoptosis assay}

The Annexin V-FITC apoptosis detection kit (BD Biosciences, San Jose, CA, USA) was used to analyze cell apoptosis, following the manufacturer's protocols. In brief, Hep-2 cells, which were transfected with siNC or 2 PRDX3 shRNAs, and normal Hep-2 cells without treatment were collected after dissociation with ethylenediaminetetraacetic acid (EDTA)-free trypsin and then washed with cold PBS. Then, the cells were re-suspended in the binding buffer with the addition of Annexin V-FITC and propidium iodide for $15 \mathrm{~min}$ of incubation in the dark. Finally, flow cytometry analysis was performed immediately thereafter on the BD FACS Calibur (BD Biosciences, San Jose, CA, USA).

\section{Cell migration}

An in vitro cell migration assay was performed in 24-well Transwell chambers (Costar, Tewksbury, MA, USA) as previously described [24]. Briefly, the upper and lower culture compartments of each well in the Transwell chambers are separated by polycarbonate membranes $(8$ $\mu \mathrm{m}$ pore size). Hep-2 cells $\left(2 \times 10^{5}\right)$ transfected in si-NC or 2 PRDX3 shRNAs in $0.1 \mathrm{ml}$ of serum-free DMEM medium were added to the upper compartment, and 0.6 $\mathrm{ml}$ of DMEM containing 10\% fetal bovine serum was placed into the lower compartment. Cells were incubated for $4 \mathrm{~h}$ at $37^{\circ} \mathrm{C}$ and stained with $0.1 \%$ crystal violet. Nonmigrating cells retained on the upper side of the membrane were removed by wiping with a cotton swab. Cells that had migrated through the membrane and had reached the underside of the membrane were counted in 4 microscopic fields using a 20×objective microscope (IX51, Olympus, Japan).

\section{Statistical analysis}

Statistical analyses were performed using SPSS 19.0 software (SPSS Inc., Chicago, IL, USA). Differences among the groups were determined by Fisher's exact test. In all patients, overall survival was defined as the time between the dates of treatment (surgery) and the dates of last follow-up or death. Survival data of patients were analyzed using the Kaplan-Meier estimation method, and the survival curves were evaluated with the log-rank test. P $<0.05$ was considered to indicate a statistically significant difference.

\section{Abbreviations}

PRDX, peroxiredoxin; LSCC, laryngeal squamous cell carcinoma; HNC, head and neck cancer; NSCLC, non-small cell lung cancer; ROS, reactive oxygen species; $\mathrm{H}_{2} \mathrm{O}_{2}$, hydrogen peroxide; TBS, tris-buffered saline; IOD, integrated optical density; BSA, bovine serum albumin;
RT-PCR, reverse transcription-polymerase chain reaction; $\mathrm{CCK}$, cell counting kit.

\section{ACKNOWLEDGMENTS}

The authors gratefully thank Ms. Lei Jiang and Ms. Xia Zhang for laboratory support, and Dr. Jinguo Li and Dr. Linlin Yang for assistance with patient recruitment.

\section{CONFLICTS OF INTEREST}

The authors declare no conflicts of interest.

\section{GRANT SUPPORT}

This work was supported by grants from the Projects of Medical and Health Technology Development Program in Shandong Province (2014WS0250), Natural science funds of Shandong Province. (ZR2014HL-079), Yantai municipal scientific and technological development project (2014WS001, 2014WS016, 2016WS010, 2016WS016), and Youth science foundation of Yantai Yuhuangding Hospital (201412).

\section{Author contributions}

$\mathrm{HZ}, \mathrm{XL}$ and XS conceived the study, JC participated in its design and coordination. $\mathrm{PZ}$ and $\mathrm{HZ}$ performed the statistical analysis. HZ, JC and XS performed clinical and surgical evaluation; LC, NL, PZ carried out the histopathological evaluation of tissues, RT-PCR, western blotting and protein extraction. XL carried out the cell line culture, transfection, cell proliferation assay, cell apoptosis assay and cell migration assay. The overall study was supervised by GL. All authors revised the manuscript critically and approved the final manuscript.

\section{REFERENCES}

1. Siegel R, Naishadham D, Jemal A. Cancer statistics, 2013. CA Cancer J Clin. 2013; 63: 11-30.

2. Xia CX, Zhu Q, Zhao HX, Yan F, Li SL, Zhang SM. Usefulness of ultrasonography in assessment of laryngeal carcinoma. Br J Radiol. 2013; 86: 20130343.

3. Markou K, Christoforidou A, Karasmanis I, Tsiropoulos G, Triaridis S, Constantinidis I, Vital V, Nikolaou A. Laryngeal cancer: epidemiological data from Northern Greece and review of the literature. Hippokratia. 2013; 17: 313-8.

4. Li L, Wang J, Gao L, Gong L. Expression of paxillin in laryngeal squamous cell carcinoma and its prognostic value. Int J Clin Exp Pathol. 2015; 8: 9232-9.

5. Jemal A, Siegel R, Ward E, Hao Y, Xu J, Thun MJ. Cancer statistics, 2009. CA Cancer J Clin. 2009; 59: 225-249. 
6. Zhao XD, Zhang W, Liang HJ, Ji WY. Overexpression of miR-155 promotes proliferation and invasion of human laryngeal squamous cell carcinoma via targeting SOCS1 and STAT3. PLoS One. 2013; 8: e56395. doi: 10.1371. Epub 2013 Feb 20.

7. Zhang H, Song X, Liu X, Zhang Q, Sun Y, Chen X, Liu C, Wang Y. Preliminary proteomic analysis of human serum from patients with laryngeal carcinoma. Eur Arch torhinolaryngol. 2012; 269:557-63.

8. Yang Y, Karakhanova S, Hartwig W, D'Haese JG, Philippov PP, Werner J, Bazhin AV. Mitochondria and Mitochondrial ROS in Cancer: Novel Targets for anticancer therapy. J Cell Physiol. 2016; 231:2570-81.

9. Song X, Xie L, Wang X, Zeng Q, Chen TC, Wang W, Song X. Temozolomide-perillyl alcohol conjugate induced reactive oxygen species accumulation contributes to its cytotoxicity against non-small cell lung cancer. Sci Rep. 2016; 6: 22762. doi: 10.1038 .

10. Rowson-Hodel AR, Berg AL, Wald JH, Hatakeyama J, VanderVorst K, Curiel DA, Leon LJ, Sweeney C, Carraway KL 3rd. Hexamethylene amiloride engages a novel reactive oxygen species- and lysosome-dependent programmed necrotic mechanism to selectively target breast cancer cells. Cancer Lett. 2016; 375: 62-72.

11. Shan Y, Guan F, Zhao X, Wang M, Chen Y, Wang Q, Feng $\mathrm{X}$. Macranthoside B induces apoptosis and autophagy via reactive oxygen species accumulation in human ovarian cancer A2780 cells. Nutr Cancer. 2016; 68: 280-9.

12. Chae HZ, Kim HJ, Kang SW, Rhee SG. Characterization of three isoforms of mammalian peroxiredoxin that reduce peroxides in the presence of thioredoxin. Diabetes Res Clin Pract. 1999, 45: 101-12.

13. Lee SS, Tsai $\mathrm{CH}$, Yu CC, Chang YC. Elevated snail expression mediates tumor progression in areca quid chewing-associated oral squamous cell carcinoma via reactive oxygen species. Plos One. 2013; 8: e67985.

14. Hu CT, Wu JR, Cheng CC, Wang S, Wang HT, Lee MC, Wang LJ, Pan SM, Chang TY, Wu WS. Reactive oxygen species-mediated PKC and integrin signaling promotes tumor progression of human hepatoma HepG2. Clin Exp Metastasis. 2011; 28: 851-63.

15. Yanagawa T1, Omura K, Harada H, Ishii T, Uwayama J, Nakaso K, Iwasa S, Koyama Y, Onizawa K, Yusa H,
Yoshida H. Peroxiredoxin I expression in tongue squamous cell carcinomas as involved in tumor recurrence. Int J Oral Maxillofac Surg. 2005; 34:915-20.

16. Ralhan R1, Desouza LV, Matta A, Chandra Tripathi S, Ghanny S, Datta Gupta S, Bahadur S, Siu KW. Discovery and verification of head-and-neck cancer biomarkers by differential protein expression analysis using iTRAQ labeling, multidimensional liquid chromatography, and tandem mass spectrometry. Mol Cell Proteomics. 2008; 7:1162-73.

17. Zhang B1, Wang K, He G, Guan X, Liu B, Liu Y, Bai Y. Polymorphisms of peroxiredoxin 1, 2 and 6 are not associated with esophageal cancer. J Cancer Res Clin Oncol. 2012; 138:621-6.

18. Beevi SS, Rasheed MH, Geetha A. Evidence of oxidative and nitrosative stress in patients with cervical squamous cell carcinoma. Clin Chim Acta. 2007; 375: 119-23.

19. Kim YS, Lee HL, Lee KB, Park JH, Chung WY, Lee KS, Sheen SS, Park KJ, Hwang SC. Nuclear factor E2-related factor 2 dependent overexpression of sulfiredoxin and peroxiredoxin III in human lung cancer. Korean J Intern Med. 2011; 26: 304-13.

20. Bookman MA. Developmental chemotherapy and management of recurrent ovarian cancer. J Clin Oncol. 2003; 21: 149s-167s.

21. Brown KK, Eriksson SE, Arner ES, Hampton MB. Mitochondrial peroxiredoxin 3 is rapidly oxidized in cells treated with isothiocyanates. Free Radic Biol Med. 2008; 45: 494-502.

22. Paleri V, Mehanna H, Wight RG. TNM classification of malignant tumours 7th edition: what's new for head and neck? Clin Otolaryngol. 2010; 35:270-2.

23. Liu XX, Zhang H, Shen XF, Liu FJ, Liu J, Wang WJ. Characteristics of testis-specific phosphoglycerate kinase 2 and its association with human sperm quality. Hum Reprod. 2016; 31: 273-9.

24. Chen Y, Quyang GL, Yi H, Li MY, Zhang PF, Li C, Li JL, Liu YF, Chen ZC, Xiao ZQ. Identification of RKIP as an invasion suppressor protein in nasopharyngeal carcinoma by proteomic analysis. J Proteome Res. 2008; 7: 5254-62. 